GEORGETOWN SCIENTIFIC RESEARCH JOURNAL
Volume Two Edition One Fall 2021

\title{
CRISPR and COVID-19: Lessons \\ Learned to Prepare for the Next \\ Pathogen
}

James M. Bond 


\title{
CRISPR and COVID-19: Lessons Learned to Prepare for the Next Pathogen
}

\author{
James M. Bond
}

Department of Science, Technology, and International Affairs, Georgetown University, Washington, D.C., United States.

E-mail: jmb516@georgetown.edu

https://doi.org/10.48091/gsr.v2i1.29

\begin{abstract}
CRISPR-Cas is a gene editing technology that can strengthen a defense countermeasure against an infectious pathogen and can heighten the attack risk of an engineered pathogen. The purpose of this report is twofold: to analyze the advantages of CRISPR for participants within a strategic environment, such as rogue, non-state attackers and defenders coordinated between nation-states and other entities, and to identify the ways in which CRISPR configures a defender's countermeasure against a biological event. In its assessment, this report utilizes the case study of COVID-19 to examine the applications of CRISPRCas systems to SARS-CoV-2. This report finds that CRISPR reduces some barriers to entry and exacerbates the possibility for malicious non-state attackers to engineer a pathogen and engender a serious biological event in the very short-term. However, key barriers to entry will continue to pose challenges to attackers comparative to defenders. In this report, "attacker" refers to non-state actors maliciously using CRISPR to engender a biological event while "defender" refers to coordinated entities responding to biological events, whether natural or deliberate. In the short- to mid-term, the use of CRISPR-Cas systems in designing a countermeasure against a biological event is to the advantage of the defender. CRISPR offers more accessible, rapid, and convenient diagnostic testing; a quick and accurate platform to identify viral vectors; and the potential for antiviral therapy. Through enactment of certain policy configurations, the comparative advantage of CRISPR may decisively shift to the defender, including in the very short-term.
\end{abstract}

Keywords: COVID-19, CRISPR, Biosecurity, Dual-Use Risk Technology

\section{Introduction}

In December of 2019, a novel coronavirus, now known as SARS-CoV-2, was identified in Wuhan, China. ${ }^{1}$ The disease caused by this virus Coronavirus disease 19 (COVID-19) - spread throughout the world and was eventually characterized as a pandemic by the World Health Organization (WHO). ${ }^{2}$ From the first cases in China to the ongoing pandemic, COVID-19 has presented major challenges to economies, governments, health systems, and communities everywhere. As of August $20^{\text {th }}, 2021$, over $200,000,000$ cases have been reported and $4,300,000$ deaths have been attributed to COVID19 , according to the WHO. ${ }^{3}$

On December 2, 2020, the first fully tested immunization was approved for use. ${ }^{4}$ Various other tested immunizations were approved across the globe shortly after. At this time, around $4,850,000,000$ vaccines have been administered, 
according to the Center for Systems Science and Engineering (CSSE) at Johns Hopkins University. ${ }^{5}$ However, waves of COVID-19 cases continue to rise in several countries, especially due to highly transmissible variants and insufficient population immunity. ${ }^{6,7}$ Furthermore, due to an immense scope of global vaccine inequity, a majority of low- and lower-middle-income countries may continue to experience surges in COVID-19 cases for years to come. ${ }^{8}$ These facts drive continued public health measures for countries, as they denote that the COVID-19 pandemic is not over.

The imperative to constrain this virus motivated countries to take innovative defense measures against COVID-19. Among these innovative applications was a gene editing technology that was discovered in the 2010s and is based on natural bacterial immune mechanisms against viruses called CRISPR-Cas systems. ${ }^{9}$ The systems are composed of two components: a guide RNA that identifies a target gene and a CRISPRassociated protein that can cut double stranded DNA, allowing for site-specific genome modification that is quick, cheap, and relatively user-friendly. ${ }^{10}$ Researchers are now faced with deciding how best to optimize the system for human applications, and the COVID-19 pandemic presented a myriad of pressing public health challenges that CRISPR could help mitigate.

Simultaneously, CRISPR gene editing technology has the potential to be abused for malicious applications to the opposite effect of aiding public health responses. Specifically, CRISPR is often touted for its comparative ease of use and efficiency in editing genes, ${ }^{11}$ which could present the opportunity for malicious attackers to genetically engineer pathogens into a biological weapon. As CRISPR reduces barriers of entry due to its low cost and ease of use, more attackers could use CRISPR to cause a deliberate biological event (DBE).

This report will analyze the advantages CRISPR holds for the attacker and defender, as well as how the technology can be leveraged to configure a countermeasure against biological events to enhance global biopreparedness. Utilizing CRISPR during the COVID-19 pandemic represents expanded use of a novel technology for a public health crisis. This report will begin by examining the risk factors that malicious non-state attackers utilizing CRISPR and other developing phenomena pose for the strategic environment. The report will then move to the application of CRISPR from three principal areas: COVID-19 diagnostic tests, pathogenic research, and the potential of antiviral therapy. Finally, the report will draw conclusions pertaining to the global health strategic environment and recommend policy to bolster global biopreparedness.

In this report, "non-state malicious attacker", or "attacker", refers to rogue groups or individuals utilizing CRISPR with malicious intent to engender a biological event. The term "biological event" refers to an outbreak of a disease caused by a pathogen and can be naturally occurring or deliberately released. ${ }^{12}$ "Defender" refers to the coordinated entities responding to a natural or deliberate biological event, including, but not limited to, governments, the scientific community, institutions, and sectors. "Biopreparedness" refers to a state of readiness a defender builds for potential biological events. ${ }^{13}$ The "strategic environment" is defined as the complex interaction of entities such as "attackers" and "defenders" amid dynamic and contradictory global forces. ${ }^{14}$ "Pathogenic surveillance regime" refers to a coordinated program to survey and curtail the infectious progression of a pathogen within a society, usually directed by a government. 


\section{Anticipating the threat of CRISPR engineered pathogens}

CRISPR-Cas systems can be utilized to delete genetic sequences, add genetic sequences, regulate expression of a phenotype, and even combine genetic sequences to produce a novel expression. ${ }^{15}$ CRISPR-Cas systems are democratized for use, as gene editing kits are commercially available from laboratory suppliers and genome engineering companies and are relatively inexpensive. Furthermore, CRISPR technologies - especially these gene editing kits - are touted for their easeof-use compared to other techniques for genomic manipulation. ${ }^{15}$ However, the same accessibility, low cost, and ease-of-use of CRISPR-Cas could greatly expand the scope of potential users. In many ways, it is a boon for positive biological applications, and research in the field has flourished due to the democratization of gene editing. In other ways, the technology could pose a biosecurity risk if applied to malicious genomic engineering, rendering it a dual use research of concern. That is, research that advances our understanding of CRISPR-Cas has the potential to be directly misapplied and pose a biological threat.

Regardless of the numerous positive applications, CRISPR could open the door for malicious non-state attackers to engineer optimized pathogenic bioweapons. Prior genomic manipulation techniques posed significant barriers of entry, requiring resources and scientific expertise for performing expensive and complex biological procedures such as constructing zincfinger arrays or delivering gene editing materials into cells. ${ }^{16,17}$ CRISPR-Cas systems circumvent some of the impediments apparent in other methods like TALENs (Transcription activatorlike effector nucleases) or zinc fingers. In fact, the concerns that the broad distribution and low cost of the technology increase the risk for deliberate misuse were stressed in multiple US Intelligence
Community threat assessments. ${ }^{18,} 19$ These advances in gene editing highlight the modern reality that the tools necessary for genetically engineering a dangerous pathogen are increasingly available, and the biosecurity risk for a DBE could be greater now than ever before. Though potential targets of maliciously released pathogens are diverse ${ }^{20}$ for practicality in this report, discussion regarding the threat of an engineered pathogen refers to that targeting humans.

Beyond the reduced barrier of entry for engineering a pathogen, the attacker may also have certain advantages in releasing that pathogen and causing a DBE. Although there may be several similarities between the defense countermeasure against either a deliberate or natural biological event, a DBE may introduce additional requirements for several factors, from responder safety to attacker attribution. ${ }^{21}$ The determination of natural or deliberate origin would presumably be made by a coordinated effort of stakeholders from intelligence communities and clinical professionals with standard criteria for assessing the biological event. ${ }^{22}$ However, upon identification of a DBE, it still may prove difficult to discern whether this intentional release is a wild-type or genetically engineered pathogen, which is crucial information for assessing pathogenesis due to environment and natural selection. That is to say, genetically engineered pathogens may experience a comparatively more rapid process of change in selecting for new genes that make the pathogen more competitive while suppressing other genes that are metabolically expensive. ${ }^{23} \mathrm{~A}$ dynamic pathogen like this may confound an efficient countermeasure from the defender. Furthermore, convoluted dimensions and numerous stakeholders in a public health response may inhibit an efficient countermeasure by the defender. A proactive release of a pathogen by an attacker is fundamentally less complicated than a reactive response by a defender. 
Understanding the complex field of genetics for beneficial human applications is a problem facing researchers in CRISPR for today. From pleiotropic effects where one gene can have multiple expressions to epigenetic effects where expression is regulated by environmental factors, a limitation of CRISPR gene editing is scientific understanding of how genes are associated with specific phenotypic expressions. After all, CRISPR is most often and easily utilized in order to silence genes to create new phenotypes rather than add genes to create new phenotypes because of these limitations in scientific understanding. Furthermore, some have cited the fact that the notable studies utilizing CRISPR are conducted by the most well-resourced and important labs, suggesting that CRISPR still requires a significant level of expertise in order to effectively operate the technology for complicated uses. ${ }^{24}$ Thus, the barriers of entry of scientific understanding and a certain level of expertise remains for attackers. As a genetically engineered pathogen is released, it may have unpredictable and unintended effects, again due to environment and natural selection. For instance, an optimized pathogen could be engineered with the intent for higher transmission and lethality and be presumed by the attacker to cause a catastrophic DBE yet perform unpredictably when released because it kills the human host too efficiently to replicate and spread. $^{24}$ This theoretical example would unintentionally underperform the attacker's goals. Furthermore, the delivery mechanism to weaponize a biological agent has classically been a limiting factor for malicious use. ${ }^{21}$ (p. 90) This too remains as a limiting barrier of entry for contemporary attackers, particularly if the desired release is within a mass population.

Some barriers of entry persist for the attacker. Even so, a release of a genetically engineered pathogen that exhibits characteristics unintended by the attacker can still be problematic for the defender. If the attacker can surmount these barriers of knowledge and delivery, while harnessing the low cost and ease of use of CRISPR, a resulting DBE may be devastating to the defender. Therefore, it is critically important to bolster global biopreparedness in both prevention and reaction strategies. For example, monitoring supply chains of CRISPR kits and dangerous pathogenic sequences may be worthwhile. However, it seems apparent that not all biological attacks may be preventable due to the lowered barriers of access to gene editing as well as the difficulty of surveillance over genetic and CRISPR materials. Thus, for the remainder of the report, there will be a primary focus on the reaction of the defender, but this is not meant to discount the need for rethought prevention strategies. In the instances of a devastating DBE, an expeditious, prepared response to contain the spread of the pathogen should be a foremost consideration.

\section{CRISPR Applications to the COVID-19 pandemic}

This report finds that CRISPR-Cas systems can be leveraged to configure an effective countermeasure against a natural or deliberate biological event, particularly in terms of rapidity and feasibility of response. In the case study of CRISPR use in the COVID-19 public health response, three principal applications prevail: diagnostics, research, and potential antiviral therapy. CRISPR diagnostic platforms can provide accessible, rapid, and convenient tests, without sacrificing standards of accuracy. CRISPR-Cas systems as a tool for research can provide an optimized platform to identify and test vectors for countermeasure targets. Finally, CRISPR-Cas antiviral therapy may provide a needed prophylactic treatment option for COVID-19 and other current and future pathogens. 


\subsection{CRISPR diagnostic platforms}

The necessity for testing to curtail the spread of pandemic- and epidemic-prone diseases is of critical importance for adequate public health. When an infectious disease begins to spread, rapid steps must be taken to track those infected in order to protect those who are not. Numerous pandemics have emerged in the past, and in every instance, robust diagnostic testing to support pathogenic surveillance was necessary. ${ }^{25}$ In the early stages of confronting pandemic diseases, the rapid development, approval, large-scale manufacturing, and deployment of reliable, fast, and accessible diagnostic tests remains of utmost priority.

Various commentators emphasized the importance of a robust pathogenic surveillance regime during the COVID-19 response: early detection, contact tracing, and isolation of infected individuals for management of contagious individuals to limit their transmission to others. Importantly, this strategy would rely heavily on diagnostic testing, particularly in early stages to prevent a major outbreak. ${ }^{26}$ In the United States, the average COVID-19 test sample-to-answer reporting time at one point during the pandemic was 4 days - far beyond what is necessary for contact tracing - in part because the supply chain did not scale up capacity for diagnostic tests and associated materials. ${ }^{27}$ Because COVID-19 could spread from individual to individual presymptomatically, symptomatically, and asymptomatically, some studies even advocated for prioritizing test accessibility, frequency, and sample-to-answer reporting time over accuracy of positive results. $^{28}$

The gold standard for diagnostic testing of SARS-CoV-2 is the real-time reversetranscription polymerase chain reaction (RTPCR) method. ${ }^{29}$ However, this method is not without its many challenges. RT-PCR tests require specialized and expensive equipment, a complex molecular laboratory, and highly trained personnel. ${ }^{30}$ Comparatively, they are laborious and expensive tests. ${ }^{31}$ Moreover, they can produce false negative results, ${ }^{32}$ may decrease in sensitivity five days after onset of symptoms, ${ }^{33}$ and are susceptible to errors in sample collection. ${ }^{34}$ Other common tests such as enzyme-linked immunosorbent assays (ELISA) and rapid antigen and antibody tests are significantly cheaper in comparison to the gold standard RT-PCR test, but they suffer in terms of accuracy and exhibit particularly low sensitivity in the onset and first few days of illness. ${ }^{35}$ Rapid antigen and antibody tests are advantageous in accessibility for point of care and low sample-toanswer reporting time (20-60 minutes), but suffer in sensitivity at $50 \%$ in comparison to RT-PCR. ${ }^{36}$ Furthermore, such an unprecedented demand for diagnostic tests amidst the COVID-19 pandemic led to a shortage in recommended testing supply. ${ }^{37}$

These limiting factors for the gold standard RT-PCR test made it difficult to scale up manufacturing to a level concordant with high demand, often resulting in test shortages and prolonged sample-to-answer reporting time. According to the Infectious Diseases Society of America, test availability and a sample-to-answer responding time within an hour are critical conditions for a positive bearing on care and disease containment. ${ }^{38}$ In many cases, diagnostic strategies for adequate surveillance did not meet standards for the containment of COVID-19. Furthermore, gold standard RT-PCR tests don't have the practicality to provide diagnostic testing to endemic regions with limited resources. Without specialized equipment, facilities, and scientists, these complex diagnostic methods become null. However, diagnostics remain critical to limit the disease spread outside of these endemic localities and prevent global or national outbreak. ${ }^{39}$ Yet, countries face both global and national disparities in relevant resource distribution, including in diagnostic tests and related 
infrastructure or intellectual property. ${ }^{40}$ The need for a SARS-CoV-2 test that was rapid, widely distributed, accessible, and accurate drove a dedicated effort to explore innovative diagnostic strategies to address the COVID-19 crisis.

A particularly innovative strategy was CRISPR diagnostics, which relies on the technology's ability to locate specific segments of viral RNA. The approach was established in 2017, and it harnesses CRISPR to quickly pinpoint and tag pathogenic RNA without RNA isolation required by RTPCR tests that adds hours to the process. ${ }^{41,}{ }^{42}$ Diagnostic testing platforms used this strategy to deliver multiplexed and accurate detection of viral presence, with the reporting mechanism being lateral flow for an easy visual readout. ${ }^{43}$ These platforms are called "SHERLOCK" (specific high-sensitivity enzymatic reporter unlocking) and "DETECTR" (DNA endonuclease-targeted CRISPR trans reporter). ${ }^{43}, 44$ Upon development, the diagnostic approach was discussed as a revolutionary method to limit disease outbreaks of numerous infectious and non-infectious diseases, but was never approved for use. ${ }^{45}$

The COVID-19 pandemic reoriented defensive efforts within the scientific community towards the common objective to end the public health crisis, as is the case for the efforts of Sherlock and Mammoth Biosciences. These companies emphasized fast, simple, and accessible diagnostic capabilities, and touted the promise of their CRISPR platform to do so, even ceding bitter patent contentions for public health. ${ }^{46}$ Thus, Sherlock and Mammoth Biosciences adapted SHERLOCK and DETECTR for SARS-CoV2. ${ }^{47}, 48$, In March 2020, Sherlock made history with the first FDA authorized use of CRISPR in a diagnostic application, and was soon followed by Mammoth. ${ }^{49}, 50$

When compared with the gold standard RTPCR tests, CRISPR diagnostic platforms were successful in offering several advantages. These advantages did not sacrifice accuracy. The SHERLOCK adaptation for SARS-CoV-2 is said to take an hour with results that are 100\% concordant with the gold standard RT-PCR tests in terms of sensitivity and specificity. ${ }^{51}$ The DETECTR adaptation for SARS-CoV-2 is said to be an even faster sample-to-answer alternative to the gold standard RT-PCR tests, yet still comparable in terms of accuracy at $95 \%$ positive predictive agreement and $100 \%$ negative predictive agreement. ${ }^{48}$ Both SHERLOCK and DETECTR exhibit accessibility with ease-of-use qualities such as visual readouts that are converted from CRISPR activity. ${ }^{52}$ Furthermore, neither test requires complicated processing through specialized equipment..$^{53}$ Because of this profound accessibility, these diagnostic platforms are also promising for important point-of-care testing for SARS-CoV-2, although they currently lack bureaucratic authorization outside of laboratory settings. ${ }^{48,54}$

These CRISPR diagnostic platforms continue to be promising with each development. Led by CRISPR co-inventor Jennifer Doudna, one research team has recently developed a CRISPR based diagnostic approach that can detect SARSCoV-2 using only a CRISPR solution and a mobile phone..$^{55}$ The tests mix a saliva sample with a chemical solution that allows the CRISPR-Cas system to identify and cut a sequence of SARS$\mathrm{CoV}-2$, then emit a glow strong enough for a smartphone to detect. ${ }^{56}$ Thus, the test is not only capable of reporting a positive or negative result, but also a quantitative estimation of the viral load by measuring the amount of SARS-CoV-2 RNA. ${ }^{57}$ Advances like these aim to bring the user interface even closer to patients and practitioners to fill the gaps in diagnostic testing by making diagnosis user-friendly, inexpensive, and portable.

CRISPR diagnostic platforms underscored notable lessons learned in how CRISPR technologies can be harnessed to aid current and 
future public health responses. CRISPR diagnostic platforms can provide tests that are more accessible in terms of ease of use and cost, rapid in terms of sample-to-answer reporting time, and convenient in terms of point-of-care, all without sacrificing specificity or sensitivity. Moreover, CRISPR's diagnostic potential to provide unprecedented accessibility is actively being discussed, including over-the-counter CRISPR-based tests for diseases such as COVID19, HPV, HIV, malaria, Zika, tuberculosis, dengue, and even cancer. ${ }^{58,59}$ CRISPR is naturally multiplexed, meaning that it has high potential for providing a test that detects multiple diseases at once. ${ }^{60}$ Clearly, CRISPR can support pathogenic surveillance regimes by providing more efficient avenues for identification of infected individuals, thereby optimizing contact tracing and disease management efforts.

These factors by which CRISPR can augment the standards of diagnostic testing are all policy relevant. CRISPR diagnostic testing is rapid, simple, and cost-effective enough to concur with a timeline relevant to public health. Whereas gold standard RT-PCR sample-to-answer reporting time delays were largely due to supply chain issues for complicated materials and procedural issues for complicated processes, CRISPR tests can help limit pathogenic spread and optimize contact tracing by offering in-clinic results. It is perhaps equally important that detection methods prioritize point of care and accessibility so that testing strategies can feasibly extend to low- and lower-middle-income countries, remote communities that are hours away from the nearest health provider, and any other locality with limited resources or lack of required equipment. This accessibility factor of CRISPR is extremely policy relevant to prevent pathogenic spread out of endemic regions by both limiting the outbreak within these regions and reducing the need to travel outside of these regions. The low $\$ 1-2$ cost per test would be feasible for consumers and national testing programs, expanding testing capacity in economic terms. ${ }^{61}$

CRISPR-based diagnostics have not been in effect for long - the first authorized use of a CRISPR test was in March 2020. This fact necessitates more studies concerning the trade-off between CRISPR diagnostic testing and alternatives like gold standard RT-PCR tests, particularly in terms of sensitivity and specificity. However, the initial application of CRISPR for diagnostic purposes in the COVID-19 pandemic is promising. The faster, more accurate, more accessible, easier, and cheaper diagnostic potential of CRISPR could improve the pathogenic surveillance of the COVID-19 pandemic and be a boon for global biopreparedness and confrontation of diseases.

\subsection{CRISPR assisted research}

The necessity for rapid research on Sars-CoV2 facilitated by CRISPR-Cas systems enabled quicker development rates for public health countermeasures against infectious pathogens. CRISPR-Cas assisted screening is a process by which researchers can inhibit certain genetic functions in order to find the equivalent of a few needles in a haystack of a complex genome. Researchers often use this CRISPR "knock-out" technique in order to identify the effect of key genes. Researchers can also elicit gain of function by inserting a genetic sequence that the DNA repair mechanisms can utilize as a template when repairing the cuts made by the Cas proteins. These CRISPR "knock-in" techniques can be utilized, for instance, to make experimental procedures more applicable to a desired target.

During the COVID-19 pandemic, these research techniques were heavily utilized to better understand SARS-CoV-2, ultimately in order to develop and test disease countermeasures. Numerous genome-wide CRISPR screening tests 
for SARS-CoV-2 were conducted in order to better understand how the virus infected human cells. ${ }^{62,63,64}$ Some of these tests sought to identify host factors required for SARS-CoV-2 entry with the intent to establish COVID-19 countermeasures that prophylactically or therapeutically targeted the human cell. Other CRISPR screening tests sought to identify viral vectors with the intent to establish potential targets on the virus to inhibit its replication. For instance, researchers discovered both key host and viral factors that modulate SARS-CoV-2 entry into a human cell like the important human ACE2 receptor or the S1/S2 boundary of the SARS$\mathrm{CoV}-2$ spike protein. ${ }^{65}$ For the purposes of this report, understanding the terminology and complex biology behind these factors is less important than understanding the use of CRISPR in discovering them. For SARS-CoV-2 research, CRISPR screening techniques were notably utilized for the background of prophylactic and therapeutic treatment strategies by identifying factors that SARS-CoV-2 relies upon for its replication.

Furthermore, researchers utilized a CRISPR "knock-in" technique to safely apply clinical evaluation to experimental trials. In one study conducted by Sun et al., researchers effectively humanized a mouse by inserting the gene for the host factor that allows SARS-CoV-2 to enter into a human cell, the ACE2 receptor. ${ }^{66}$ By eliciting a gain of function outcome that allowed a mouse to become infected by COVID-19, these researchers created a tool that could be used to evaluate the effectiveness of COVID-19 vaccines and therapeutic treatments in clinical trials on nonhuman subjects. The mouse could then be treated with a vaccine, therapy, or drug that could not yet be ethically tried on human subjects. In the need for rapid development and evaluation of humantargeted prophylactic and therapeutic countermeasures to a pathogen, this is a crucial step to optimize procedure.

This research is the necessary background to continue developing and evaluating COVID-19 therapeutic, vaccine, and drug candidates in clinical trials. The development of these countermeasures remains crucial as nations must continue to cope with COVID-19 cases. Beyond the application of COVID-19, CRISPR can be utilized to identify factors upon which any pathogen relies for replication with these experimental techniques. In fact, researchers are now identifying viral and host factors to target for other critical diseases — such as HIV or malaria - and discussing the potential of novel countermeasures for these pathogens as well. ${ }^{67,68}$ CRISPR-Cas systems are clearly a promising research tool that can provide an efficient and accurate platform to identify and test viral vectors for needed antiviral therapeutic, vaccine, and drug targets. This tool can be utilized to optimize the configuration of a countermeasure against an emerging pathogen and mitigate its pathogenesis.

\subsection{CRISPR therapeutic treatment}

CRISPR therapy can potentially provide an important countermeasure opportunity for COVID-19, as well as the ability to reconfigure treatment for other persistent and emergent diseases. Since December 2020, vaccines have been approved and rolled-out across the world in an effort to control the pandemic. ${ }^{69}$ However, the mutation rate of SARS-CoV-2 is relatively high, and each variant poses new challenges. Human behavior, motivation, and culture continue to be essential for effective pandemic recovery. Given broad societal challenges such as vaccine hesitancy and vaccine inequity, as well as the fact that vaccinated people can still be infected and spread COVID-19, the pandemic will persist globally. ${ }^{70}$, ${ }^{71}$ As the world has seen, an outbreak of COVID19 anywhere threatens to increase the case rate 
everywhere. Thus, there is a persistent need for COVID-19 treatment options as the pandemic continues. This need can potentially be filled by CRISPR therapeutic treatment for SARS-CoV-2.

Applying CRISPR-Cas systems as a countermeasure to COVID-19 has demonstrated promising initial evaluations, although the novel therapeutic option has received comparatively less attention than other therapeutic options. ${ }^{72}$ One group of Stanford-based CRISPR researchers have developed a CRISPR facilitated therapeutic approach called prophylactic antiviral CRISPR in human cells (PAC-MAN) that can effectively degrade RNA from SARS-CoV-2 in human cells. ${ }^{73}$ A significant challenge for the Stanford team is finding a delivery mechanism for the PACMAN approach, most effectively targeting epithelial cells in the lung where the virus inflicts the most damage. If this barrier is overcome, the team believes that the PAC-MAN approach can be utilized as a countermeasure to all coronaviruses and emerging variants of SARS-CoV-2. In the same study, the approach was found to demonstrate effectiveness in degrading RNA from influenza in human cells.

Another group of CRISPR researchers utilized a highly reprogrammable CRISPR-Cas system to target SARS-CoV-2 transcripts that code for specific proteins like the spike protein. ${ }^{74}$ As mentioned, the spike protein on coronaviruses was identified by CRISPR knock-out techniques to be integral for latching onto and infecting the human cell. Ultimately, the reprogrammable CRISPRCas system demonstrated a high degree of effectiveness, with greater than $98 \%$ efficiency in silencing these regions of viral transcript. Notably, the group expressed concern for emerging variants of SARS-CoV-2 and the propensity of SARS$\mathrm{CoV}-2$ to escape from host immunity. The reprogrammable nature of this CRISPR approach is a vital countermeasure for emerging strains of SARS-CoV-2, but also for the system's adaptability to future emerging pathogens. Either the PAC-MAN approach or the reprogrammable CRISPR-Cas system can effectively deactivate SARS-CoV-2 replication cycles.

It should be noted that CRISPR-Cas therapeutic treatment is subject to significant ethical considerations. CRISPR-Cas systems recently brought discussion of gene therapy back into the limelight after tragic setbacks related to other prior gene therapy techniques. ${ }^{75}$ However, opportunities associated with CRISPR-Cas systems are not without ethical concerns as well. This is especially true regarding CRISPR germline therapy that causes genetic changes at an early age in all cells, therefore driving through generations via inheritance. Somatic gene therapy through CRISPR, on the other hand, causes genetic changes in only certain cells, but still some commentators remain skeptical and stipulate that ethical distinctions between somatic editing and the more controversial germline editing are not as clear cut as they seem. ${ }^{76}$ Furthermore, due to research and development costs, CRISPR gene therapy is likely to be restrictively expensive to lower income individuals. ${ }^{77}$ There is also significant concern for limitations to CRISPR like off-target effects - where the system cuts and replaces an unintended sequence and has unpredictable effects. Other limitations include DNA-Damage toxicity, where the editing may trigger effects like early cell death, or immunotoxicity, where the cells may build immunity against the therapeutic treatment. ${ }^{78}$ These limitations are especially of concern if the therapeutic target is the human cell.

CRISPR-Cas systems are known to be naturally occurring in bacteria with the purpose of deleting RNA from an infectious virus that hijacks cell functions. These systems can be therapeutically leveraged for somatic gene editing in human cells for the same purpose. Beyond the potential of these systems for this pandemic, 
CRISPR-Cas systems can be applied to persistent pathogens like HIV. ${ }^{79}$ Before broad application of CRISPR therapies to any disease, careful ethical considerations and clinical trials must be carried out. Thus, CRISPR antiviral therapies have not yet been clinically applied as a countermeasure against COVID-19. ${ }^{80}$ Nonetheless, the pandemic persists and there remains great potential: CRISPR-Cas systems can provide a therapeutic treatment for SARS-CoV-2 and emerging variants, setting the stage for an important reprogrammable countermeasure for future pathogens.

\section{The role of CRISPR in the strategic environment}

CRISPR democratizes gene editing in terms of ease-of-use, cost, and commercial accessibility. It is therefore possible for malicious non-state attackers to engineer a dangerous pathogen and cause a DBE. Some barriers to access remain for the attacker, including scientific knowledge in the field of genetics and mechanism of delivering an engineered pathogen. Nonetheless, the first-mover advantage is prominent as it is far less complicated to release a pathogen than to contain it. Unintended effects caused by a lack of scientific knowledge when engineering a pathogen may be irrelevant and still drive disease through society. Further, the complexity of an efficient and coordinated defense response with varied stakeholders contributes to containment difficulties. This comparative ease of release relative to containment is a key asset for the attacker and gives the CRISPR advantage to the attacker in the very short-term engineering phase and immediately following release.

In the countermeasure response to a pathogen, efficiency and rapidity are key to containment. This report finds that CRISPR can be leveraged to rapidly configure this defense countermeasure. CRISPR diagnostic tests offer certain advantages over gold standard RT-PCR tests, including accessibility, sample-to-answer reporting time, and convenience. These tests are of critical importance for a pathogenic surveillance regime to efficiently and rapidly contain the spread. CRISPR tests are a key asset for the defender - especially in initial outbreak stages - and can be leveraged further to enhance the defense advantage of CRISPR in the short term. CRISPR offers a platform to identify and test viral vectors, aiding the configuration of countermeasures like vaccines and treatments. This efficient platform is to the advantage of the defender in the short- to midterm. Finally, CRISPR offers the potential development of therapeutic treatment options for the defender's long-term countermeasure strategy since diseases aren't just eradicated. CRISPR can also test the effectiveness of these long-term therapeutic treatments.

Therefore, this report concludes that the decreased barrier to access for malicious actors to engineer a pathogen renders CRISPR more advantageous to a malicious, non-state actor in the very short-term. However, the remaining barriers for the attacker and the CRISPR defense applications against said pathogen suggest a net positive for the defender in the short- to mid-term and long-term response. The short- to mid-term response refers to the direct response to a biological event that can last months to a few years after first cases are identified; the long-term response refers to the several year- to decade-long biological preparation efforts from the defender. With increased research and development (R\&D), applied use, and normalization of CRISPR, this net positive can only improve for the defender. Through development of policies that optimize CRISPR for rapid defense applications, the comparative advantage of CRISPR may decisively shift from the attacker to the defender in the very short-term as well. 


\section{Policy recommendations}

Accordingly, this report recommends four policy changes: 1 . R\&D of CRISPR to break down limitations and build up social norms, 2. The creation of an international platform to promote information sharing, 3. Investment in a manufacturing sector for ready CRISPR diagnostic testing, and 4. Deployment of CRISPR diagnostic tests to endemic regions upon outbreak. These policy recommendations are not intended to be comprehensive. Rather the emphasis of this report is to identify some lessons learned from the applications of CRISPR during the COVID-19 pandemic as a prompt for a rethought biopreparedness strategy for an evolving global health strategic environment.

CRISPR-Cas systems have several technical limitations that include possible off-target effects, immunotoxicity, and DNA-Damage toxicity. These limitations engender social hesitancy due to reasonable ethical concerns, ultimately curtailing the defender's ability to configure a prepared and rapid public health response to a pathogen. In order for CRISPR to be applied to biopreparedness and biosafety on a needed broader scale, limitations and associated hesitancy must be addressed. Thus, the first recommendation in this report is increased $\mathrm{R} \& \mathrm{D}$ funding and efforts to break down limitations regarding CRISPR. Mounting use of the technology after limitations are addressed will build social norms around CRISPR and mitigate public hesitancy for safe applications.

One notable enabling factor for the recent applications of CRISPR was the decision to put patents aside to aid the effort to contain COVID19. ${ }^{44}$ This decision seems to signify that lessened resources devoted to court fights along with the information sharing for a common cause allowed for more efficient development and application of CRISPR. Scientific research builds upon other scientific research, and with an ever-intensifying threat of infectious disease, the scientific community must accelerate R\&D of CRISPR for defense applications. Thus, this report also recommends an international platform for information sharing surrounding altruistic CRISPR research efforts for biopreparedness, coordinated by the World Health Organization.

A primary intervention for a defender preventing a widespread outbreak of disease is diagnostic testing to support an efficient pathogenic surveillance regime. This report therefore recommends incentivizing national private sectors to create new manufacturing capacity for ready CRISPR diagnostic tests for priority pathogens. Furthermore, this report recommends that nations deploy these portable CRISPR diagnostic tests to endemic regions upon outbreak. COVID-19 has demonstrated that an outbreak anywhere is a threat everywhere, and the low-cost, portable, and easy-to-use characteristics of CRISPR diagnostic tests could be leveraged to rapidly contain pathogens.

\section{Conclusion}

The strategic environment for biosecurity is more unstable than ever before. CRISPR further exacerbates an already heightened threat of infectious disease by reducing barriers to entry for genetically engineered pathogens. At the same time, CRISPR is a remarkable tool for defense against either a natural or deliberate biological event. Applied use in the COVID-19 pandemic afforded several lessons learned for how CRISPR can help support pathogenic surveillance regimes, research, and countermeasure development. CRISPR must first overcome technical limitations and social hesitancy, then be broadly applied to biopreparedness strategies like CRISPR diagnostic tests on stand-by. This type of rethought approach is necessary to fully leverage the technology to the advantage of the defender in a transformed biosecurity strategic environment. 
Before COVID-19, commentators warned of the emergence of a novel, pandemic-level pathogen. When it arrived, we were not prepared to contain it. Moreover, the interplay between an attacker, a defender, and CRISPR within the contemporary strategic environment does not exist in a vacuum, and there are also several concerning humandriven trends that exacerbate risk: global warming, ${ }^{81-84}$ permafrost thaw, ${ }^{85,86}$ climate-related migration, ${ }^{87}$ deforestation, ${ }^{88,89}$ and loss of biodiversity. ${ }^{90}$ The critical need for biopreparedness strategies to counteract the increased risk of biological events, whether deliberate or natural, is greater than ever before. When the next pathogen arrives, will we have learned to leverage CRISPR for a prepared and rapid response? Our survival may depend on it.

\section{Acknowledgments}

This report is produced as part of the $2021 \mathrm{Lisa}$ J. Raines "Grand Challenge" Undergraduate Research Fellowship. Throughout the research and writing of this report I have received a great deal of support and assistance.

I would first like to thank my faculty sponsor, Dr. Carol Kuntz, whose guidance was invaluable for integrating a higher degree of precision into my research design, overcoming research barriers, and writing the report for a specified audience. Our meetings were refreshing, clarifying, and ultimately brought this and future work to a higher level.

I would also like to thank the Lisa J. Raines Fellowship and Georgetown University's Center for Research and Fellowships for affording me this opportunity. The program design prioritized interdisciplinary collaboration between a wide array of peer-led projects, as well as provided expert support and insight. This was integral to success in the final product of my research.

\section{References}

1. Zhu, H., Wei, L. \& Niu, P. The novel coronavirus outbreak in Wuhan, China. Global Health Research and Policy. 2020; 5 (6). https://doi.org/10.1186/s41256-020-00135-6

2. WHO Director-General's opening remarks at the media briefing on COVID-19 - 11 March 2020. Who.int. https://www.who.int/directorgeneral/speeches/detail/who-director-general-sopening-remarks-at-the-media-briefing-on-covid19---11-march-2020. Published 11 March 2020. Accessed 18 August 2021.

3. WHO Coronavirus (COVID-19) Dashboard. Who.int. https://covid19.who.int/. Published 11 March 2020. Updated 20 August 2021. Accessed 20 August 2021.

4. Ledford, H., Cyranoski, D. \& Van Noorden, R.. Nature. 2020; 588, 205-206. doi: https://doi.org/10.1038/d41586-020-03441-8

5. COVID-19 Dashboard by the Center for Systems Science and Engineering (CSSE) at Johns Hopkins University (JHU). Coronavirus.jhu.edu. https://coronavirus.jhu.edu/map.html. Published 11 March 2020. Updated 20 August 2021. Accessed 20 August 2021.

6. Mahase, E.. Delta variant: What is happening with transmission, hospital admissions, and restrictions? BMJ. 2021; 373 (1513). doi: https://doi.org/10.1136/bmj.n1513

7. CDC COVID-19 Vaccine Breakthrough Case Investigations Team. COVID-19 Vaccine Breakthrough Infections Reported to CDC United States, January 1-April 30, 2021. MMWR Morb Mortal Wkly Rep. 2021;70(21):792-793.

Published 2021 May 28. doi:10.15585/mmwr.mm7021e3

8. Asundi, A., O'Leary, C., Bhadelia, N.. Global COVID-19 vaccine inequity: The scope, the impact, and the challenges. Cell Host $\mathcal{E}^{3}$ Microbe. 2021; 29 (7), 1036-1039. doi: https://doi.org/10.1016/j.chom.2021.06.007

9. Ledford, H., \& Callaway, E.. Pioneers of revolutionary CRISPR gene editing win chemistry Nobel. Nature. 2020; 586, 346-347. doi: https://doi.org/10.1038/d41586-020-02765-9 
10. Redman M, King A, Watson C, King D. What is CRISPR/Cas9?. Arch Dis Child Educ Pract Ed. 2016;101(4):213-215. doi: 10.1136/archdischild2016-310459

11. CRISPR-based technologies for cell biology. Nat Cell Biol. 2019; 21, (1463). https://doi.org/10.1038/s41556-019-0434-y

12. Katz, R., Graeden, E., Kerr, J., The complexity of biological events. The Lancet. 2018; 6 (2), 136137. doi: https://doi.org/10.1016/S2214109X(17)30494-1

13. Thompson, T. L.. Biopreparedness and biosecurity. Encyclopedia of health communication, SAGE Publications, Inc.. 2014; 1, 114-116. https://www.doi.org/10.4135/9781483346427.n43

14. U.S. Department of the Navy. Chapter 1, the Strategic Environment in MCDP 1-1. Published 1997. Accessed August 18, 2021. https:/www.globalsecurity.org/military/library/poli cy/usmc/mcdp/1-1/mcdp1-1 chap1.pdf

15. Ceasar, A., Rajan, A., Prykhozhij, S., Berman, J., Ignacimuthu. Insert, remove or replace: A highly advanced genome editing system using CRISPR/Cas9. Biochimica et Biophysica Acta (BBA) - Molecular Cell Research. 2016; 1863 (9), 23332344. doi: https://doi.org/10.1016/j.bbamcr.2016.06.009

16. Aken, J., and Hammond, E.. Genetic Engineering and Biological Weapons. Science and Society. 2003; 4 (Special Issue) 57-60.

17. Gaj, T., Sirk, S. J., Shui, S., \& Liu, J. (2016). Genome-Editing Technologies: Principles and Applications. Cold Spring Harbor Perspectives in Biology, 8(12), a023754. https://doi.org/10.1101/cshperspect.a023754

18. U.S. Intelligence Community. Worldwide Threat Assessment of the US Intelligence Community. Published 2016. Accessed August 18, 2021. https://www.dni.gov/files/documents/SASC Uncl assified 2016 ATA SFR FINAL.pdf

19. U.S. Intelligence Community. Worldwide Threat Assessment of the US Intelligence Community. Published 2018. Accessed August 18, 2021. https://www.dni.gov/files/documents/Newsroom/ Testimonies/2018-ATA---Unclassified-SSCI.pdf 20. Ryan J. Biosecurity and Bioterrorism. ButterworthHeinemann; 2016.
21. Katz R, Graeden E, Abe K, Attal-Juncqua A, Boyce MR, Eaneff S. Mapping stakeholders and policies in response to deliberate biological events. Heliyon. 2018; 4 (12). doi: 10.1016/j.heliyon.2018.e01091

22. Grunow, R., Finke, E.-J.. A procedure for differentiating between the intentional release of biological warfare agents and natural outbreaks of disease: its use in analyzing the tularemia outbreak in Kosovo in 1999 and 2000. Clinical Microbiology and Infection. 2002; 8 (8), 510-521. doi: https://doi.org/10.1046/j.1469-0691.2002.00524.x

23. Vogel, K. M., \& Ouagrham-Gormley, S. B. (2018). Anticipating emerging biotechnology threats: A case study of CRISPR. Politics and the Life Sciences, 37(2), 203-219. https://doi.org/10.1017/pls.2018.21

24. Pope SM. Impact of Gene Editing Tools, Like CRISPR/Cas9, on the Public Health Response to Disease Outbreaks. Disaster Medicine and Public Health Preparedness. 2017; 11 (2):155-159. doi: 10.1017/dmp.2016.123

25. Piret J, Boivin G. Pandemics Throughout History. Front Microbiol. 2021; 11. doi: 10.3389/fmicb.2020.631736

26. Hassell, J.. Testing early, testing late: four countries' approaches to COVID-19 testing compared. Ourworldindata.org. Published May 19, 2020. Accessed August 18, 2021.

https://ourworldindata.org/covid-testing-us-ukkorea-italy\#licence

27. Botti-Lodovico, Y., M.P.P., Rosenberg, E., M.D., and Sabeti, P., D.Phil., M.D.. Testing in a

Pandemic - Improving Access, Coordination, and Prioritization. N EnglJ Med. 2021; 384, 197-199. doi: 10.1056/NEJMp2025173

28. Larremore, D., Wilder, B., Lester, E., Shehata, S., Burke, J., Hay, J., Tambe, M., Mina, M., Parker, J.. Test sensitivity is secondary to frequency and turnaround time for COVID-19 surveillance. Sci Adv. 2021; 7 (1). doi: 10.1126/sciadv.abd5393

29. Oliveira BA, Oliveira LC, Sabino EC, Okay TS. SARS-CoV-2 and the COVID-19 disease: a mini review on diagnostic methods. Rev Inst Med Trop Sao Paulo. 2020; 62, 44. doi:10.1590/S16789946202062044 
30. Rao, G., Agarwal, A., and Baturad, D.. Testing times in Coronavirus disease (COVID-19): A tale of two nations. Med J Armed Forces India. 2020; 76 (3), 243-249. doi: 10.1016/j.mjafi.2020.05.014

31. Du, Z., PhD, Pandey, A., PhD, Bai, Y., PhD, Fitzpatrick, M., PhD, Chinazzi, M., PhD, Pastore y Piontti, A., PhD, et al. Comparative costeffectiveness of SARS-CoV-2 testing strategies in the USA: a modelling study. The Lancet. 2021; 6

(3) 184-191. doi: https://doi.org/10.1016/S24682667(21)00002-5

32. Chen Z, Li Y, Wu B, Hou Y, Bao J, Deng X. A Patient with COVID-19 Presenting a FalseNegative Reverse Transcriptase Polymerase Chain Reaction Result. Korean J Radiol. 2020; 21 (5), 623-624. doi: https://doi.org/10.3348/kjr.2020.0195

33. Kucirka, L., MD, PhD, Lauer, S., PhD*, Laeyendecker, O., PhD, MBA, Boon, D., PhD, Lessler, J., PhD. Variation in False-Negative Rate of Reverse Transcriptase Polymerase Chain Reaction-Based SARS-CoV-2 Tests by Time Since Exposure. Annals of Internal Medicine. 2020; 173, 262-267. doi: https://doi.org/10.7326/M20-1495

34. Hernández-Péreza, J-M., Martín-Gonzálezb, E., Pino-Yanesb, M.. Strengths and weaknesses of the diagnostic tests for SARS-CoV-2 infection. Medicina Clinica (English Edition). 2020; 155 (10): 464-465. doi: 10.1016/j.medcle.2020.05.025

35. Hernández-Pérez, J. M., Martín-González, E., \& Pino-Yanes, M. (2020). Strengths and weaknesses of the diagnostic tests for SARS-CoV-2 infection. Medicina Clinica (English Edition), 155(10), 464465. https://doi.org/10.1016/j.medcle.2020.05.025

Lambert-Niclot, S., Cuffel, A., Pape, S. L., Vauloup-Fellous, C., Morand-Joubert, L., RoqueAfonso, A.-M., Goff, J. L., \& Delaugerre, C. (2020). Evaluation of a Rapid Diagnostic Assay for Detection of SARS-CoV-2 Antigen in Nasopharyngeal Swabs. Journal of Clinical Microbiology. https://doi.org/10.1128/JCM.0097720

37. Barra GB, Santa Rita TH, Mesquita PG, Jácomo RH, Nery LFA. Overcoming Supply Shortage for SARS-CoV-2 Detection by RT-qPCR. Genes
(Basel). 2021;12 (1): 90. doi:

10.3390/genes 12010090

38. Caliendo, A., Gilbert, D., Ginocchio, C., Hanson, K., May, L., Quinn, T., Tenover, F., Alland, D., Blaschke, A., Bonomo, R., Carroll, K., Ferraro, M-J., Hirschhorn, L., Joseph, W.P., Karchmer, T., MacIntyre, A., Reller, L., Jackson, A., Clinical Infectious Diseases. 2013; 57 (3): 139-170. doi: https://doi.org/10.1093/cid/cit578

39. Kelly-Cirino CD, Nkengasong J, Kettler H, et al. Importance of diagnostics in epidemic and pandemic preparedness. BMJ Global Health. 2019; 4. doi: http://dx.doi.org/10.1136/bmjgh-2018$\underline{001179 .}$

40. Engel N, Wachter K, Pai M, et al. Addressing the challenges of diagnostics demand and supply: insights from an online global health discussion platform. BMJ Global Health. 2016; 1. doi: http://dx.doi.org/10.1136/bmjgh-2016-000132

41. J. S. Gootenberg, O. O. Abudayyeh, J. W. Lee, P. Essletzbichler, A. J. Dy, J. Joung, V. Verdine, N. Donghia, N. M. Daringer, C. A. Freije, C. Myhrvold, R. P. Bhattacharyya, J. Livny, A. Regev, E. V. Koonin, D. T. Hung, P. C. Sabeti, J. J. Collins, F. Zhang, Nucleic acid detection with CRISPR-Cas13a/C2c2. Science. 2017; 356, (3663): 438-442. doi: 10.1126/science.aam9321

42. Garcia-Venzor A, Rueda-Zarazua B, MarquezGarcia E, Maldonado V, Moncada-Morales A, Olivera H, Lopez I, Zuñiga J and MelendezZajgla J. SARS-CoV-2 Direct Detection Without RNA Isolation With Loop-Mediated Isothermal Amplification (LAMP) and CRISPR-Cas12. Front. Med. 2021; 8. doi: 10.3389/fmed.2021.627679

43. Gootenberg, J., Abudayyeh, O., Kellner, M., Joung, J., Collins, J., Zhang, F.. Multiplexed and portable nucleic acid detection platform with Cas13, Cas12a, and Csm6. Science. 2018; 360 (6387): 439-444. doi: 10.1126/science.aaq0179

44. Chen, J., Ma, E., Harrington, L., Da Costa, M., Tian, X., Palefsky, J., Doudna, J.. CRISPRCas12a target binding unleashes indiscriminate single-stranded DNase activity. Science. 2018; 360, (6387): 436-439. doi: 10.1126/science.aar6245 
45. Chertow, D.. Next-generation diagnostics with CRISPR. Science. 2018; 360 (6387): 381-382. doi: 10.1126/science.aat4982

46. Isaacson, W.. CRISPR rivals put patents aside to help in fight against Covid-19. STAT News. Published March 3, 2021. Accessed August 19, 2021.

https://www.statnews.com/2021/03/03/crisprrivals-put-patents-aside-fight-against-covid-19/

47. Joung J, Ladha A, Saito M, et al. Point-of-care testing for COVID-19 using SHERLOCK diagnostics. Preprint. medRxiv. 2020;

2020.05.04.20091231. doi: 10.1101/2020.05.04.20091231

48. Broughton, J.P., Deng, X., Yu, G. et al. CRISPRCas12-based detection of SARS-CoV-2. Nat Biotechnol. 2020; 38: 870-874. doi: https://doi.org/10.1038/s41587-020-0513-4

49. Guglielmi, G.. First CRISPR test for the coronavirus approved in the United States. Nature. 2020. doi: https://doi.org/10.1038/d41586-020$\underline{01402-9}$

50. U.S. Food and Drug Administration. Accelerated emergency use authorization (EUA) summary SARS-CoV-2 RNA DETECTR assay. Published 2020. Accessed August 18, 2021. https://www.fda.gov/media/139937/download

51. Khan, W., Barney, R., Tsongalis, G.. CRISPRcas13 enzymology rapidly detects SARS-CoV-2 fragments in a clinical setting. Preprint. medRxiv. 2020. doi: https://doi.org/10.1101/2020.12.17.20228593

52. Sherlock Biosciences. High Throughput SHERLOCK CRISPR SARS-CoV-2 Test. White Paper. BioRxiv. 2020.

https://sherlock.bio/wpcontent/uploads/2021/01/High Througput SHE RLOCK SARS CoV2 test Jan2021.pdf

53. Tsang, J. and LaManna, C. Open Sharing During COVID-19: CRISPR-Based Detection Tools. The CRISPR Journal. 2020; 3 (3). doi: https://doi.org/10.1089/crispr.2020.0030

54. U.S. Food and Drug Administration. Fact sheet for healthcare providers: Sherlock ${ }^{\mathrm{TM}}$ CRISPR SARS-CoV-2 kit. Published January 142021. Accessed August 18, 2021. https://www.fda.gov/media/137745/download
55. Fozouni, P., Son, S., de León Derby, M., Knott, G., Gray, C., D’Ambrosio, M., Zhao, C., Switz, N., Renuka Kumar, G., Stephens, S., Boehm, D., Tsou, C-L., Shu, J., Bhuiya, A., Armstrong, M., Harris, A., Chen, P-Y., Osterloh, J., MeyerFranke, A., Joehnk, B., Walcott, K., Sil, A., Langelier, C., Pollard, K., Crawford, E., Puschnik, A., Phelps, M., Kistler, A., DeRisi, J., Doudna, J., Fletcher, D., Ott, M.. Amplification-free detection of SARS-CoV-2 with CRISPR-Cas13a and mobile phone microscopy. Cell. 2020; 184 (2). doi: https://doi.org/10.1016/j.cell.2020.12.001

56. Ning, B., Yu, T., Zhang, S., Huang, Z., Tian, D., Lin, Z., Niu, A., Golden, N., Hensley, K., Threeton, B., Lyon, C., Yin, X-M., Roy, C., Saba N., Rappaport, J., Wei, Q., and Hul, T.. A smartphone-read ultrasensitive and quantitative saliva test for COVID-19. Science Advances. 2021; 7, (2). doi: 10.1126/sciadv.abe3703

57. Manke, K.. New CRISPR-based COVID-19 test uses smartphone cameras to spot virus RNA.

Berkeley Newus. 2020.

https://news.berkeley.edu/story jump/new-crisprbased-covid-19-test-uses-smartphone-cameras-tospot-virus-rna/

58. Béchard, D.. Over-the-Counter Cancer Tests? Stanford Magazine. 2019.

https://stanfordmag.org/contents/over-thecounter-cancer-tests-mammoth-biosciences

59. Harmon Courage, K.. The race to make coronavirus testing as easy as a pregnancy test. $V o x$ Magazine. 2020. https://www.vox.com/2020/6/3/21277347/covid19-testing-at-home-accuracy

60. Ackerman, C.M., Myhrvold, C., Thakku, S.G. et al. Massively multiplexed nucleic acid detection with Cas13. Nature. 2020; 582: 277-282. doi: https://doi.org/10.1038/s41586-020-2279-8

61. Lucia, C., Federico, P.-B., Carla Alejandra, G.. An ultrasensitive, rapid, and portable coronavirus SARS-CoV-2 sequence detection method based on CRISPR-Cas12. BioRxiv. Preprint. 2020. doi: https://doi.org/10.1101/2020.02.29.971127

62. Yaron, T., Heaton, B., Levy, T., Johnson, J., Jordan, T., Cohen, B., Kerelsky, A., Lin, T-Y., Liberatore, K., Bulaon, D., Kastenhuber, E., Mercadante, M., et al. The FDA-approved drug 
Alectinib compromises SARS-CoV-2

nucleocapsid phosphorylation and inhibits viral infection in vitro. bioRxiv. Preprint.

2020.08.14.251207. doi:

https://doi.org/10.1101/2020.08.14.251207

63. Hoffmann, H., Sánchez-Rivera, F., Schneider, W., Luna, J., Soto-Feliciano, Y., Ashbrook, A., Le Pen, J., Leal, A., Ricardo-Lax, I., Michailidis, E., Hao, Y., Stenzel, A., Peace, A., Zuber, J., Allis, C., Lowe, S., MacDonald, M., Poirier, J., Rice, C. Cell Host \& Microbe. 2021; 29 (2): 267-280. doi: https://doi.org/10.1016/j.chom.2020.12.009

64.

Baggen, J., Persoons, L., Vanstreels, E. et al. Genome-wide CRISPR screening identifies TMEM106B as a proviral host factor for SARSCoV-2. Nat Genet. 2021; 53: 435-444. doi: https://doi.org/10.1038/s41588-021-00805-2

65. Zhu, Y., Feng, F., Hu, G. et al. A genome-wide CRISPR screen identifies host factors that regulate SARS-CoV-2 entry. Nat Commun. 2021; 12: 961. doi: https://doi.org/10.1038/s41467-021-21213-4 Sun SH, Chen Q, Gu HJ, Yang G, Wang YX, Huang XY, Liu SS, Zhang NN, Li XF, Xiong R, Guo Y, Deng YQ, Huang WJ, Liu Q, Liu QM, Shen YL, Zhou Y, Yang X, Zhao TY, Fan CF, Zhou YS, Qin CF, Wang YC. A Mouse Model of SARS-CoV-2 Infection and Pathogenesis. Cell Host Microbe. 2020; 28 (1): 124-133. doi: 10.1016/j.chom.2020.05.020

67. Krasnopolsky, S., Kuzmina, A., Taube, R. Genome-wide CRISPR knockout screen identifies ZNF304 as a silencer of HIV transcription that promotes viral latency. PLOS Pathogens. 2020. doi: https://doi.org/10.1371/journal.ppat.1008834 Vijayan, K., Arang, N., Wei, L., Morrison, R., Geiger, R., Parks, K., Lewis, A., Mast, F., Douglass, A., Kain, H., Aitchison, J., Johnson, J., Aderem, A., Kaushansky, A.. A genome-wide CRISPR-Cas9 screen identifies CENPJ as a host regulator of altered microtubule organization during Plasmodium liver infection. Preprint. BioRxiv. 2021; doi: https://doi.org/10.1101/2020.08.31.275867

69. Tregoning, J.S., Flight, K.E., Higham, S.L. et al. Progress of the COVID-19 vaccine effort: viruses, vaccines and variants versus efficacy, effectiveness and escape. Nat Rev Immunol. 2021. doi: https://doi.org/10.1038/s41577-021-00592-1

70. Shah, H.. COVID-19 recovery: science isn't enough to save us. Nature. 2021; 591: 503. doi: https://doi.org/10.1038/d41586-021-00731-7

71. Griffin, S. (2021). Covid-19: Fully vaccinated people can carry as much delta virus as unvaccinated people, data indicate. $B M J, 374$, n2074. https://doi.org/10.1136/bmj.n2074

72. Lotfi, M. and Rezaei, N. CRISPR/Cas13: A potential therapeutic option of COVID-19. Biomedicine E Pharmacotherapy. 2020; 131. doi: https://doi.org/10.1016/j.biopha.2020.110738

73. Abbott TR, Dhamdhere G, Liu Y, et al. Development of CRISPR as an Antiviral Strategy to Combat SARS-CoV-2 and Influenza. Cell. 2020; 181 (4): 865-876. doi: 10.1016/j.cell.2020.04.020

74. Fareh, M., Zhao, W., Hu, W. et al. Reprogrammed CRISPR-Cas13b suppresses SARS-CoV-2 replication and circumvents its mutational escape through mismatch tolerance. Nat Commun. 2021; 12: 4270. doi: https://doi.org/10.1038/s41467-021-24577-9

75. Sibbald B. Death but one unintended consequence of gene-therapy trial. CMAJ. 2001; 164 (11): 1612.

76. Johnston, J. (2020). Shaping the CRISPR GeneEditing Debate: Questions About Enhancement and Germline Modification. Perspectives in Biology and Medicine, 63(1), 141-154.

https://doi.org/10.1353/pbm.2020.0011

77. Sherkow JS. CRISPR, Patents, and the Public Health. Yale J Biol Med. 2017; 90 (4): 667-672.

78. Uddin F, Rudin CM, Sen T. CRISPR Gene Therapy: Applications, Limitations, and Implications for the Future. Front Oncol. 2020; 10: 1387. doi:10.3389/fonc.2020.01387

79. Panfil, A., Green, P., and Yoder, K.. CRISPR Genome Editing Applied to the Pathogenic Retrovirus HTLV-1. Front. Cell. Infect. Microbiol. 2020. doi: https://doi.org/10.3389/fcimb.2020.580371

80. Kumar, P., Malik, Y. S., Ganesh, B., Rahangdale, S., Saurabh, S., Natesan, S., Srivastava, A., Sharun, K., Yatoo, Mohd. I., Tiwari, R., Singh, R. K., \& Dhama, K. (2020). CRISPR-Cas System: An Approach With Potentials for COVID-19 
Diagnosis and Therapeutics. Frontiers in Cellular and Infection Microbiology, 10, 639. https://doi.org/10.3389/fcimb.2020.576875

81. Curseu, D., Popa, M., SirbuIoan Stoian, D.. Potential Impact of Climate Change on Pandemic Influenza Risk. Global Warming. 2010; 643-657. doi: https://doi.org/10.1007/978-1-4419-10172_45

82. Thomas, MB. Epidemics on the move: Climate change and infectious disease. PLoS Biol. 2020; 18 (11). doi: https://doi.org/10.1371/journal.pbio.3001013

83. Patz, J. A., Githeko, A. K., McCarty, J. P., Hussein, S., Confalonieri, U., de Wet, N.. Climate change and infectious diseases in Climate change and human health: Risks and responses. 2003; 103-132. https://citeseerx.ist.psu.edu/viewdoc/download?doi $=10 \cdot 1 \cdot 1 \cdot 1054.1929 \&$ \&rep $=$ rep1\&type $=$ pdf

84. Martens WJ, Niessen LW, Rotmans J, Jetten TH, McMichael AJ. Potential impact of global climate change on malaria risk. Environ Health Perspect. 1995; 103 (5): 458-464. doi: 10.1289/ehp. 95103458

85. Gross, M.. Permafrost thaw releases problems. Current Biology. 2019; 29 (2), 39-41. https://doi.org/10.1016/j.cub.2018.12.045
86. Stella, E., Mari, L., Gabrieli, J. et al. Permafrost dynamics and the risk of anthrax transmission: a modelling study. Sci Rep. 2020; 10. https://doi.org/10.1038/s41598-020-72440-6

87. McMichael, C.. Climate change-related migration and infectious disease. Virulence. 2015; 6 (6). https://doi.org/10.1080/21505594.2015.1021539

88. Morris, A., Guégan, J-F., Andreou, D., Marsollier, L., Carolan, K., Le Croller, M., Sanhueza, D., Gozlan, R.. Deforestation-driven food-web collapse linked to emerging tropical infectious disease, Mycobacterium ulcerans. Science Advances. 2016; 2 (12). doi: 10.1126/sciadv.1600387

89. Henrique Ellwanger, J. and Artur B. Chies, J.. Beyond diversity loss and climate change: Impacts of Amazon deforestation on infectious diseases and public health. An. Acad. Bras. Ciênc. 2020; 92 (01). doi: https://doi.org/10.1590/0001$\underline{3765202020191375}$

90. Wood, C., Lafferty, K., DeLeo, G., Young, H., Hudson, P., Kuris, A.. Does biodiversity protect humans against infectious disease? Ecology. 2014; 95 (4), 817-832. doi: https://doi.org/10.1890/131041.1 


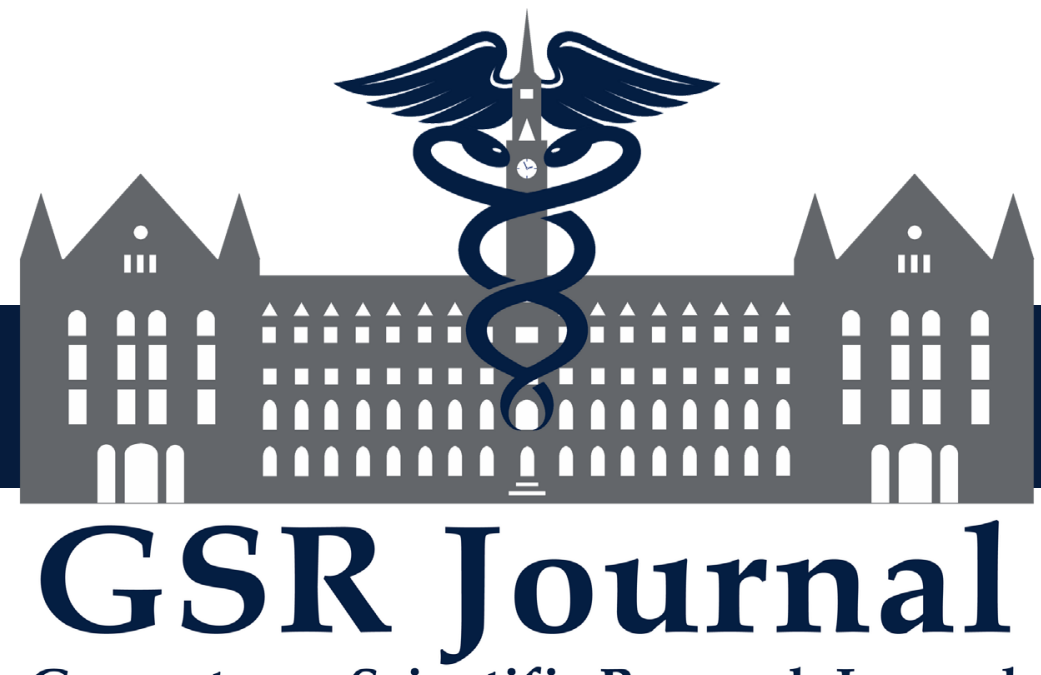

Georgetown Scientific Research Journal 